\title{
Nanoparticle Precipitation in Reverse Microemulsions: Particle Formation Dynamics and Tailoring of Particle Size Distributions
}

\author{
Björn Niemann ${ }^{1}$, Peter Veit ${ }^{2}$ and Kai Sundmacher ${ }^{1,3, *}$ \\ ${ }^{1}$ Max Planck Institute for Dynamics of Complex Technical Systems, \\ Sandtorstr. 1, D-39106 Magdeburg, Germany \\ ${ }^{2}$ Institute for Experimental Physics, Otto-von-Guericke University Magdeburg, \\ Universitätsplatz 2, D-39106 Magdeburg, Germany \\ ${ }^{3}$ Process Systems Engineering, Otto-von-Guericke University Magdeburg, \\ Universitätsplatz 2, D-39106 Magdeburg, Germany \\ ${ }^{*}$ Corresponding author: Professor Dr.-Ing. Kai Sundmacher, \\ Max Planck Institute for Dynamics of Complex Technical Systems, \\ Sandtorstr. 1, D-39106 Magdeburg, Germany \\ Phone: +49 3916110 350, Fax: +49 3916110 353, \\ E-mail: sundmacher@mpi-magdeburg.mpg.de
}




\section{Supporting Information}

\section{S.1 Particle analysis and statistics}

For particle analysis different TEM techniques were used. Particle sizes, particle morphologies and the particle size distributions were obtained from photographs taken with a CCD camera (Keen view, Olympus Soft Imaging Solutions GmbH) at a Philips CM200 TEM (200 kV, bright field condition), where the nanoparticles were placed on a gold grid with 100 $\mu \mathrm{m}$ mesh size and a film coverage consisting of chloroform dissolved Mowital F40 which is a formaldehyde-acetal (TEM pictures partially conducted by the Institute for Electron Microscopy and Analytics in Halle/ Saale, Germany with a completely similar TEM). Gold grids are chemically inert and therefore any influence of the grid on the product can be excluded. Cryo-TEM pictures (conducted by the analytics institute AQura GmbH, Hanau (Germany) with a LEO 912ab TEM from Zeiss NTS GmbH) were taken to prove the long time stability of the particles and to show that the particles are not agglomerated or influenced by the preparation of the samples on the gold grids (see figure S1). Additionally HR-TEM (High Resolution) pictures were taken with the Philips TEM mentioned above to investigate the crystal structure, which appeared to be mono-crystalline (see figure S2).

A very fast sample preparation had to be guaranteed for an accurate analysis of the particle formation dynamics inside the reactor. Therefore, a $0.5 \mathrm{ml}$ sample was taken from the reactor by a syringe with a long pipe located on the opposite side of the feed pipe. Two redundant gold grids were used for each sample and each was fast covered drop-wise with $200 \mu$ l of the solution. Excess solution was removed by filter paper where the gold grids were placed on. The time needed for this procedure was around 60 seconds, which is very short compared to the total processing time of 2 hours. Afterwards the gold grids were placed on a special support, which ensures that the chloroform dissolved Mowital film is not being destroyed, and 
put inside a vacuum dryer for more than one hour to remove remaining water and cyclohexane. After drying the gold grids were washed with a 2:1 acetone/ water solution to remove the surfactant and again dried inside the vacuum dryer for more than one hour. A comparison of particles prepared by this procedure with the particles obtained by the CryoTEM analysis did not show any significant differences of the particle sizes and their morphology. The particles on Cryo-TEM images were not specially prepared with washing and drying steps. They were obtained by direct freezing of the complete solution, thus any influence of the sample preparation can be excluded.

The particle size distributions were derived by measuring each single particle on the TEM pictures. Spherical particles are characterized by their diameter, rectangular plates by the length of the two sides and the thickness and hexagonal plates by the longest and shortest diagonal and the thickness. For each particle the volume has been calculated and from this volume an equivalent sphere diameter is obtained, which represents the particle size in all shown figures in this work. The thickness of the plates was derived from particles lying on the side (see figure 4c). The mean thickness for each experiment was calculated from these values and has been used for the volume calculation of all particles for one particle size distribution. An average of ca. 1000 particles was measured individually within the software tool analySISpro (Soft Imaging Solutions $\mathrm{GmbH}$ ) for the derivation of each particle size distribution (6 - 7 per experiment). This high number of particles ensures statistical independence of the results. 

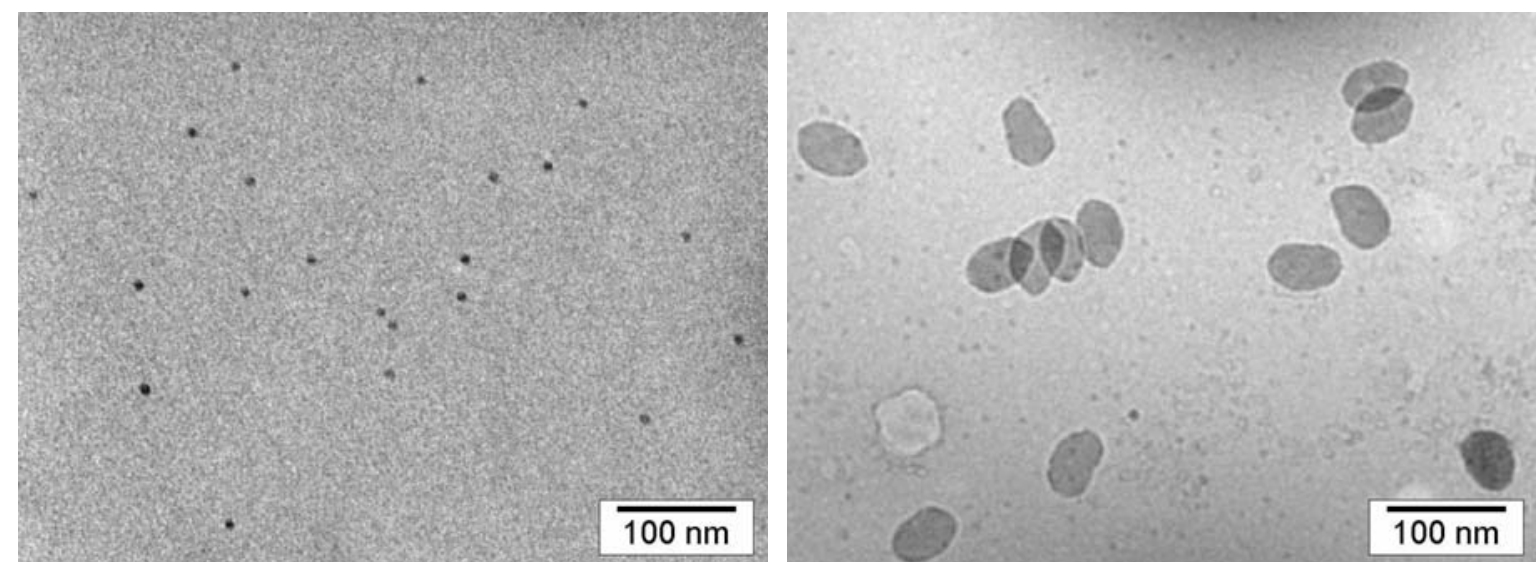

Figure S1: Cryo-TEM pictures from particles produced at $\Delta c_{0}=0 \mathrm{~mol} / \mathrm{l}(\mathrm{E} 1$, left $)$ and $\Delta c_{0}=$ $0.095 \mathrm{~mol} / \mathrm{l}$ (E3, right).

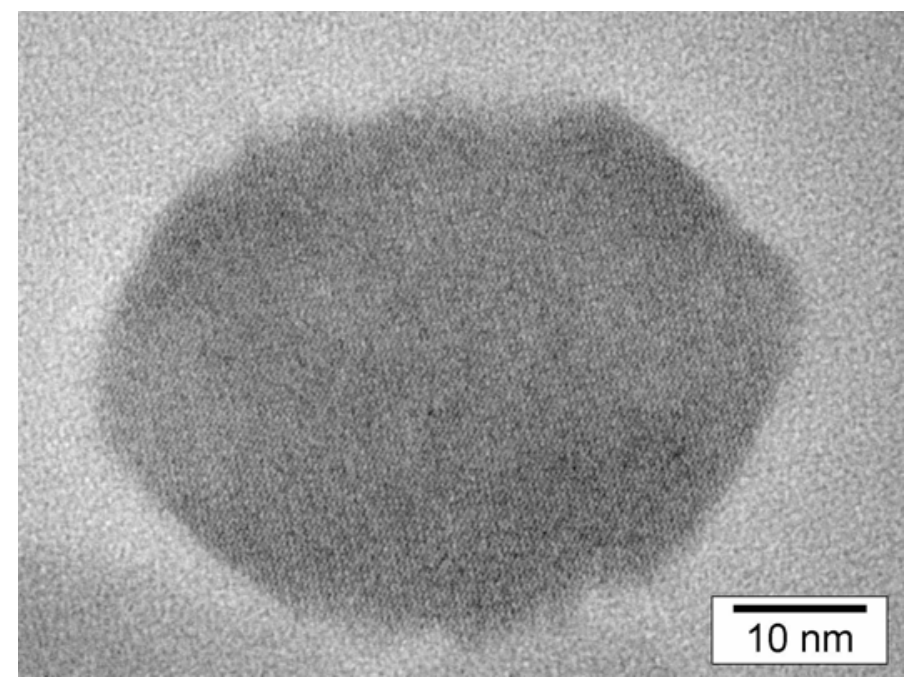

Figure S2: HR-TEM picture of a $\mathrm{BaSO}_{4}$ nanoparticle (E3).

In table S1 some statistical parameters for each experiment are given in order to prove the statistical independence of the obtained results on the number of analyzed particles. 
Table S1: Statistical parameters

\begin{tabular}{|c|c|c|c|}
\hline Exp. & Analyzed PSD* & $\varnothing$ Particles/PSD & TEM pictures \\
\hline \hline E1 & 7 & 988 & 90 \\
\hline E2 & 7 & 910 & 227 \\
\hline E3 & 6 & 467 & 183 \\
\hline E4 & 6 & 1702 & 248 \\
\hline E5 & 6 & 323 & 210 \\
\hline E6 & 7 & 1138 & 140 \\
\hline E7 & 7 & 1453 & 199 \\
\hline$\varnothing$ total & 6.6 & 997 & 185 \\
\hline
\end{tabular}

* PSD: Particle Size Distribution

S.2 Particle shape analysis by tipping stage examinations

The proof that the particles have a plate-like morphology is shown in figure S3. It shows particles from experiment E3 from different angles.

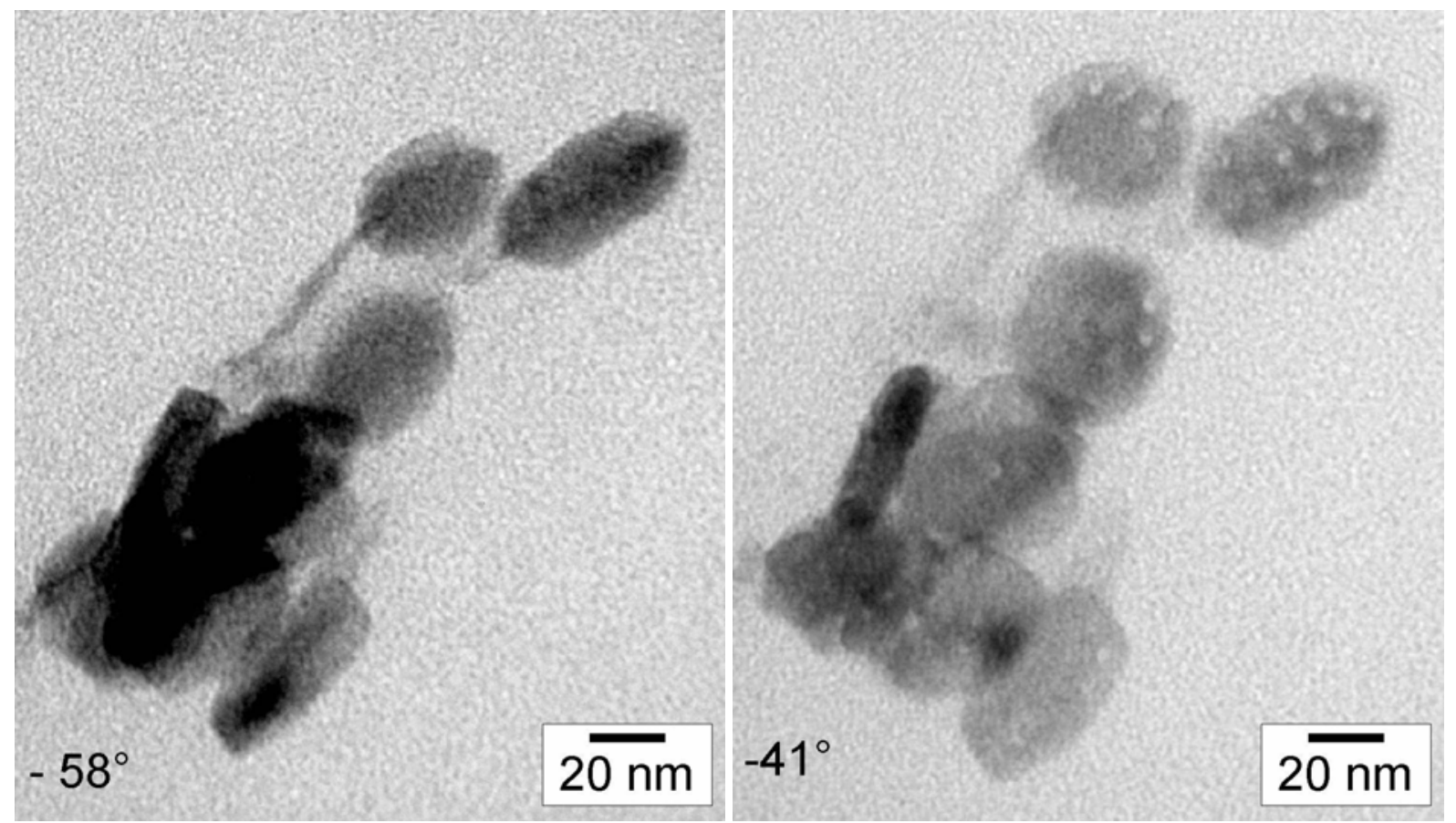




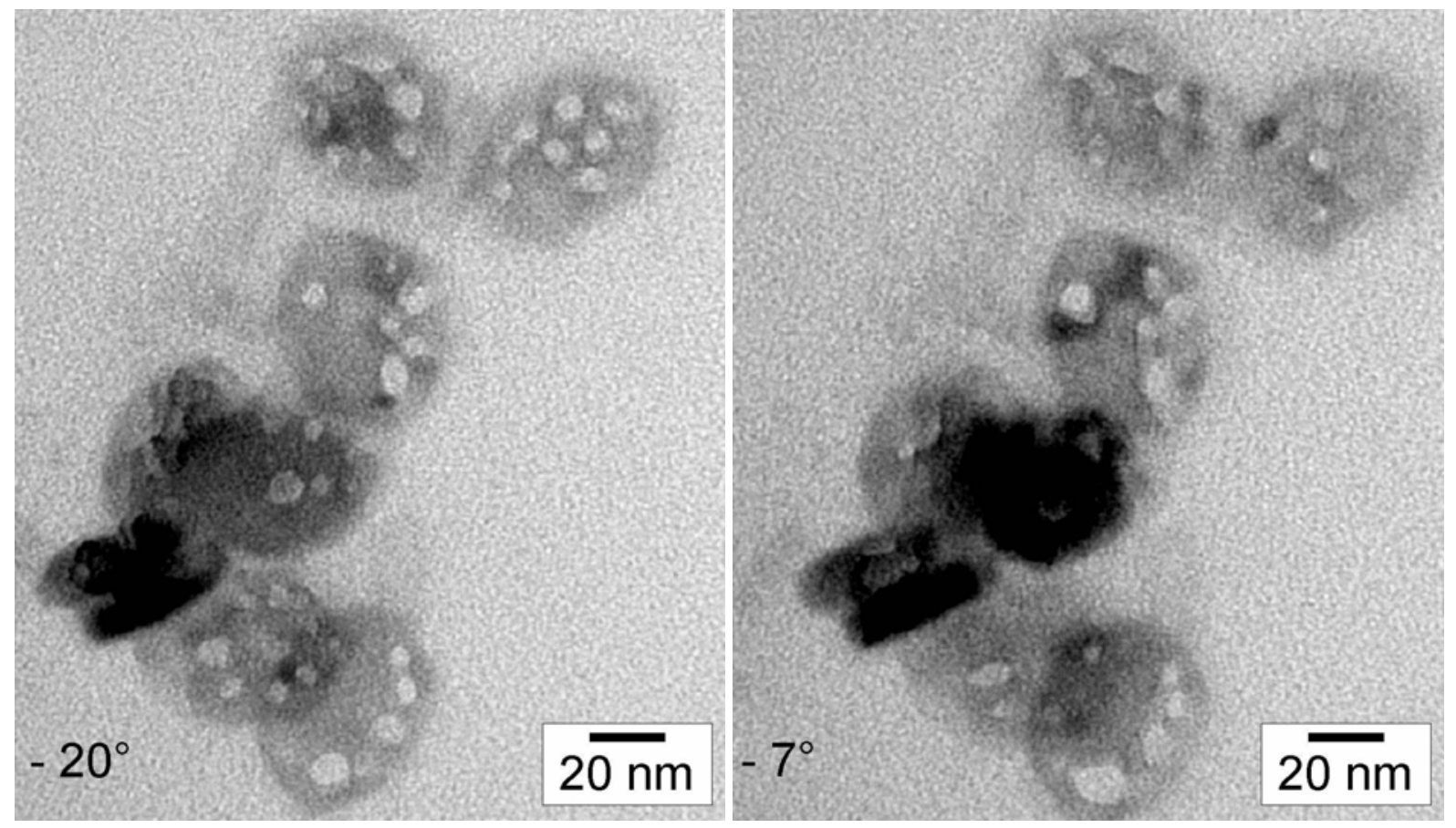

Figure S3: Tipping stage examinations of the barium sulfate nanoparticles from E3 which prove that the particles have a plate-like morphology. The holes in the particles result from long time radiation of the sample with electrons. 